\title{
SCIDoC
}

International Journal of Clinical Dermatology \& Research (IJCDR)

ISSN: 2332-2977

\section{Dupilumab: Friend or Foe to Alopecia Areata?}

Eingun James Song ${ }^{*}$, Audrey J Wong, BS

North Sound Dermatology, Mill Creek, WA, USA.

\section{Introduction}

Dupilumab is an interleukin (IL)-4 receptor alpha antagonist approved for the treatment of moderate-to-severe atopic dermatitis. Since its approval in 2017, several case reports of dupilumab both causing and treating alopecia areata in patients with atopic dermatitis have surfaced in the literature. In the cases of new-onset or reactivation of alopecia areata, no predisposing risk factors have been identified [1-5]. In the case reports of dupilumab treating alopecia areata, all three patients had a childhood history of atopic dermatitis with a concomitant diagnosis of alopecia areata months to years before starting treatment with dupilumab [6-8]. We report the fourth published case of alopecia areata being successfully treated with dupilumab.

\section{Case Presentation}

A 69-year old Caucasian female with a history of childhood atopic dermatitis and hypothyroidism presented to our clinic with an acute onset of diffuse, widespread scalp and eyebrow hair loss. Initial lab work was unremarkable, including a normal thyroid function panel, ferritin and vitamin D levels. Biopsy was done to rule out telogen effluvium and confirmed the diagnosis of diffuse alopecia areata.

Patient was subsequently treated with topical steroids, intralesional triamcinolone, topical minoxidil, fexofenadine, ezetimibesimvastatin, high dose prednisone and methotrexate with no evidence of hair regrowth. It was decided to initiate treatment with dupilumab given that she also had concomitant atopic dermatitis. Patient received subcutaneous dupilumab $600 \mathrm{mg}$ at week 0 , then $300 \mathrm{mg}$ every two weeks thereafter. At her seven-month follow up, patient had achieved $100 \%$ regrowth of her hair and complete clearance of her atopic dermatitis. Patient decided to stop treatment at that time because of her out of out-of-pocket costs and persistent conjunctivitis that was not responding to artificial tears and steroid drops. Two months after her last dose of dupilumab, patient reported diffuse hair shedding which prompted her to restart dupilumab along with methotrexate $10 \mathrm{mg} /$ wk. At her three- month follow up which was the last time patient was seen as of today's writing, she continued to lose hair which was confirmed by a positive hair pull test in all four quadrants and has decided to stop all medical treatment.

\section{Discussion}

The duality of dupilumab as a treatment and the cause of alopecia areata speaks to the complex nature of its immunopathogenesis. Alopecia areata is a well-known comorbidity of atopic dermatitis and may share a common Thelper 2 (Th2) immune pathway [9]. This could potentially explain why the use of dupilumab which inhibits Th2 signaling can be a therapeutic option for these patients.

Interestingly, alopecia areata has historically been considered a Th1 immune mediated disease through the effects of interferongamma[10-12]. However, pre-clinical studies have also shown aberrant Th2 signalingin animal models [13] and even a mixed Th1, Th2 and Th17 immune response in others [14]. Therefore, the traditional binary classification of Th1 and Th2 mediated disease may be too simplistic and the immunopathogenesis is more heterogenous than once thought. More work needs to be done to better understand the immunopathogenesis of alopecia areata and to predict appropriate candidates for dupilumab treatment. Clinical trials are currently underway and will hopefully provide more insight into this in the near future (ClinicalTrials. Gov NCT02018042).

What makes our case unique is that this patient's atopic dermatitis was relatively mild compared to the other three reported cases. Therefore, it doesn't appear that atopic dermatitis severity can predict potential responders. In addition, our patient had dramatic regrowth of her hair just four months into treatment. Given that the onset of her hair loss was just a few weeks before starting treatment, one can wonder if aggressive treatment early on will lead to a more favorable response compared to those with longstanding disease, as has been the case with janus kinase (JAK) inhibitors. Lastly, our case demonstrates that the clinical benefit

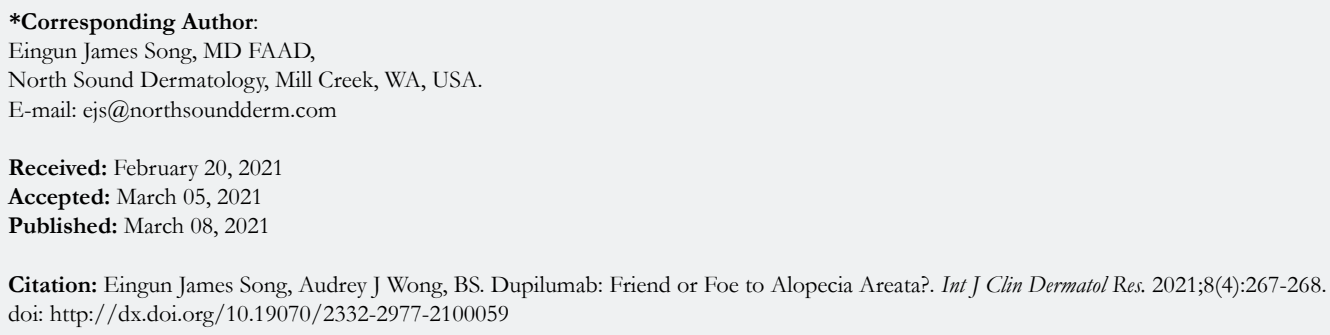


of dupilumab in alopecia areata is not sustained once treatment is stopped, as our patient relapsed two months after her last dose which mirrors the experience with JAK inhibitors [15]. Furthermore, our patient failed to recapture her previous efficacy after 3 months of restarting dupilumab.

\section{Conclusion}

In conclusion, we present the fourth reported case of dupilumab successfully treating severe widespread alopecia areata in a patient with concomitant atopic dermatitis. We also provide additional patient characteristics that may be used to better predict potential responders to dupilumab treatment, as well as further evidence that treatment must be continued even after achieving complete hair regrowth given the high likelihood of recurrence and that recapture of efficacy may not be possible for some patients who stop therapy.

\section{References}

[1]. Flanagan K, Sperling L, Lin J. Drug-induced alopecia after dupilumab therapy. JAAD Case Rep. 2018 Dec 6; 5(1): 54-56. PMID: 30560185.

[2]. Chung J, Slaught CL, Simpson EL. Alopecia areata in 2 patients treated with dupilumab: New onset and worsening. JAAD Case Rep. 2019 Jul 30; 5(8): 643-645. PMID: 31388527.

[3]. Carnicle JM, Hendricks AJ, Shi VY. Reactivation of Alopecia Areata After Dupilumab Therapy for Atopic Dermatitis. Dermatitis. 2019 Aug 21. PMID: 31441782

[4]. Yazdanyar S, Jemec GBE. Alopecia Areata After Treatment with Dupilumab. Dermatitis. 2019 Mar/Apr; 30(2): 175-176. PMID: 30829800.

[5]. Mitchell K, Levitt J. Alopecia areata after dupilumab for atopic dermatitis. JAAD Case Rep. 2018 Jan 16; 4(2): 143-144. PMID: 29387767.
[6]. Darrigade AS, Legrand A, Andreu N, Jacquemin C, Boniface K, Taïeb A, et al. Dual efficacy of dupilumab in a patient with concomitant atopic dermatitis and alopecia areata. Br J Dermatol. 2018 Aug; 179(2): 534-536. PMID: 29710431.

[7]. Penzi LR, Yasuda M, Manatis-Lornell A, Hagigeorges D, Senna MM. Hair Regrowth in a Patient With Long-standing Alopecia Totalis and Atopic Dermatitis Treated With Dupilumab. JAMA Dermatol. 2018 Nov 1; 154(11): 1358-1360. PMID: 30304403.

[8]. Uchida H, Kamata M, Watanabe A, Agematsu A, Nagata M, Fukaya S, et al. Dupilumab Improved Alopecia Areata in a Patient with Atopic Dermatitis: A Case Report. Acta Derm Venereol. 2019 Jun 1; 99(7): 675-676. PMID: 30938821.

[9]. Andersen YM, Egeberg A, Gislason GH, Skov L, Thyssen JP. Autoimmune diseases in adults with atopic dermatitis. J Am Acad Dermatol. 2017 Feb; 76(2): 274-280.e1. PMID: 27742171.

[10]. Andersen YM, Egeberg A, Gislason GH, Skov L, Thyssen JP. Autoimmune diseases in adults with atopic dermatitis. J Am Acad Dermatol. 2017 Feb; 76(2): 274-280.e1. PMID: 27742171.

[11]. Kuwano Y, Fujimoto M, Watanabe R, Ishiura N, Nakashima H, Ohno Y, et al. Serum chemokine profiles in patients with alopecia areata. Br J Dermatol. 2007 Sep; 157(3): 466-73. PMID: 17489976.

[12]. Sadeghi S, Sanati MH, Taghizadeh M, Mansouri P, Jadali Z. Study of Th1/ Th2 balance in peripheral blood mononuclear cells of patients with alopecia areata. Acta Microbiol Immunol Hung. 2015 Sep; 62(3): 275-85. PMID: 26551570.

[13]. Malik K, Guttman-Yassky E. Cytokine Targeted Therapeutics for Alopecia Areata: Lessons from Atopic Dermatitis and Other Inflammatory Skin Diseases. J Investig Dermatol Symp Proc. 2018 Jan; 19(1): S62-S64. PMID: 29273110.

[14]. Suárez-Farińas M, Ungar B, Noda S, Shroff A, Mansouri Y, Fuentes-Duculan J, et al. Alopecia areata profiling shows TH1, TH2, and IL-23 cytokine activation without parallel TH17/TH22 skewing. J Allergy Clin Immunol. 2015 Nov; 136(5): 1277-87. PMID: 26316095.

[15]. Phan K, Sebaratnam DF. JAK inhibitors for alopecia areata: a systematic review and meta-analysis. J Eur Acad Dermatol Venereol. 2019 May; 33(5): 850-856. PMID: 30762909. 